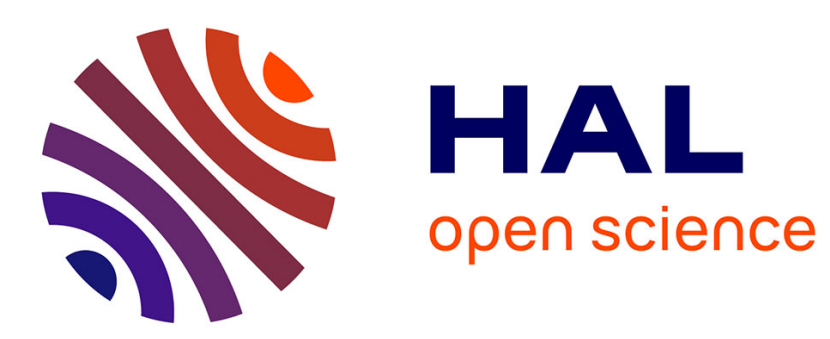

\title{
A molecular model for flow induced crystallization of polymers
}

\author{
Arnaud Poitou, Amine Ammar
}

\section{To cite this version:}

Arnaud Poitou, Amine Ammar. A molecular model for flow induced crystallization of polymers. Macromolecular Symposia, 2002, Flow-Induced Crystallization of Polymers 185 (1), pp.243-255. 10.1002/1521-3900(200208)185:13.0.CO;2-S . hal-01004850

\section{HAL Id: hal-01004850 https://hal.science/hal-01004850}

Submitted on 29 Jan 2017

HAL is a multi-disciplinary open access archive for the deposit and dissemination of scientific research documents, whether they are published or not. The documents may come from teaching and research institutions in France or abroad, or from public or private research centers.
L'archive ouverte pluridisciplinaire HAL, est destinée au dépôt et à la diffusion de documents scientifiques de niveau recherche, publiés ou non, émanant des établissements d'enseignement et de recherche français ou étrangers, des laboratoires publics ou privés. 


\title{
A Molecular Model for Flow Induced Crystallization
}

\section{of Polymers}

\author{
Arnaud POITOU, Amine AMMAR
}

LMT, ENS de Cachan, 61 Avenue du Président Wilson 94235 Cachan CEDEX - France

poitou@lmt.ens-cachan.fr and ammar@lmt.ens-cachan.fr

Summary: We present in this paper a thermodynamic model for flow induced crystallization of a thermoplastic. The thermomechanical framework (generalized standard materials) allows us to couple in a very natural way the kinetics of crystallization with the mechanical history experienced by the thermoplastic ${ }^{[1]}$. In describing the viscoelastic properties of the polymer with a molecular theory, we obtain a model for flow-induced crystallization that couples the chain conformation to the kinetics of crystallization. This model intends to be valid both for shearing and elongation. We present the equations for two cases: Maxwell and Pom-Pom constitutive equations. We finally illustrate our model with injection molding simulations achieved with a dedicated Finite Element code.

\section{Introduction}

The aim of this paper is to present a model that couples the mechanical history experienced by a polymer to its kinetics of crystallization. This model is obtained in the thermodynamic framework of so called "standard materials" that is extended here to molecular theories. In most existing theories (see ${ }^{[2][3][4]}$ for example), flow induced crystallization is modelled with two independent steps. In a first one, kinetics laws are parameterised by flow conditions (shear or elongation) and independently or in a second step the mechanical materials properties (viscosity, relaxation time ...) are parameterised by the degree of crystallinity. Our point of view is similar to the one developed by McHughs and co-workers ${ }^{[5]}$. We believe that it is possible to model flow induced crystallization in the framework of irreversible thermodynamics. This means in particular that the two-abovementioned steps are in fact not independent (in analogy with Onsager reciprocal relations).

\section{Notation}

$\mathrm{M}$ tonsorial notation $\left(\mathrm{M}_{\mathrm{ij}}\right)$

$\underline{\underline{M}}: \underline{\underline{N}}$ trace of the matricial product $\left(\operatorname{Tr}(\underline{\underline{M}} \cdot \underline{\underline{N}})\right.$ or $\mathrm{M}_{\mathrm{ij}} \mathrm{N}_{\mathrm{ij}}$ )

$\mathrm{t}$ intrinsic time

$\mathrm{d} / \mathrm{dt}$ time material derivative

$\partial / \partial \mathrm{t}$ time partial derivative 
$\delta / \delta \mathrm{t} \quad$ time upper convective derivative

$\widetilde{D} / \widetilde{D} t$ general notation for a convective derivative

$X$ thermodynamic internal variable

$Y$ thermodynamic dual variable

$\phi \quad$ intrinsic dissipation

$\Psi \quad$ free energy (indices " $c$ " and "a" are relative to crystalline and amorphous phase)

$\varphi$ * dissipation pseudopotential (index " $N$ " refers to quiescent (natural)

crystallization and index "M" stands for mechanical dissipation)

$\rho_{0} \quad$ density

$\underline{\underline{\tau}} \quad$ extra stress tensor

$\underline{\underline{D}} \quad$ strain rate tensor $\left(D_{i j}=\left(\mathrm{v}_{\mathrm{i}, \mathrm{j}}+\mathrm{v}_{\mathrm{j}, \mathrm{i}}\right) / 2\right)$

$n, \chi \quad$ kinetic parameters

$T$ temperature

$L \quad$ latent heat

$x \quad$ degree of crystallinity

$x_{\infty} \quad$ ultimate degree of crystallinity

$\alpha \quad$ relative crystallinity

$f \quad$ intrinsic crystallinity

$\beta$ dual variable of relative crystallinity

$\Lambda \quad$ dual variable of intrinsic crystallinity

$\underline{c}$ conformation tensor

$\underline{z}$ dual variable of conformation tensor

$G \quad$ elastic shear modulus

$\underline{\underline{\varepsilon}} \quad \quad$ elastic strain tensor for infinite strains

$\underline{\underline{Y}}$ dual variable of elastic strain tensor

$\mathrm{m}, \mathrm{K}$ power-law behaviour materials constants

$\eta \quad$ viscosity

$\theta \quad$ relaxation time

$v K \quad$ number of chains per unit volume multiplied by dumbbell stiffness

$\underline{v} \quad$ velocity field

$G_{0}, \phi_{\mathrm{b}}$ material parameter of the pompom model

$\lambda \quad$ chain stretch

$\underline{\underline{S}} \quad$ chain orientation tensor

$\mu \quad$ dual variable of chain stretch

y dual variable of chain orientation tensor

$\theta_{s} \quad$ stretch relaxation time

$\theta_{\mathrm{b}} \quad$ backbone relaxation time

\section{Generalized standard materials:}

The standard material formalism has been extensively used in solids mechanics to describe elasticity, plasticity, damage as well as many different coupled phenomena. We give here a new extension of this formalism, which enables us to account for molecular parameters (conformation tensor, tube orientation or extension ...) in finite strains.

The main idea of this formalism is that two potentials are required to describe the 
behaviour of a material. The first one is a thermodynamic potential (specific Gibbs free energy $\psi$ for example), which allows us to quantify the ability of the material to store energy. The second one is called a pseudo-potential, which allows us to quantify the ability of the material to dissipate energy. The general procedure is the following:

Step 1: Identify which materials parameters $\underline{X}^{1}$ describe the storage of energy. Then quantify this energy, (i.e. give a mathematical expression for $\psi(X)$ ) and calculate the dual variable $\underline{Y}=\rho_{0} \partial \psi / \partial \underline{X}$.

Step 2: Identify a material derivative $\widetilde{D} / \widetilde{D} t$. This derivative is chosen so that for reversible processes (i.e. for short experimental time scales compared to materials relaxation times), $\widetilde{D} \underline{X} / \widetilde{D} t=\underline{0}$. Then, if we write the specific dissipation $\phi=\tau: \underline{\underline{D}}-\underline{Y} \cdot d \underline{X} / d t$ and we note that this dissipation must be zero as soon as the process is reversible (i.e. as soon as $\widetilde{D} \underline{X} / \widetilde{D} t=\underline{0}$ ), we obtain an expression for the extra stress tensor $\underline{\underline{\underline{\tau}}}$

Step 3: Identify dissipation pseudopotential $\varphi^{*}=\varphi^{*}(Y)$ such that the evolution equations write $\widetilde{D} \underline{X} / \widetilde{D} t=-\partial \varphi^{*} / \partial \underline{Y}^{2}$. In order to satisfy the second law of thermodynamics (Clausius Duhem inequality), this potential is assumed to be convex such that $\varphi^{*}(Y=0)=0$. The derivation of this pseudopotential is not always obvious, but from known constitutive relations it is usually possible to find it out.

This formalism allows us to extend the mechanical constitutive relation and to account for different coupling phenomena like induced crystallization.

\section{Induced crystallization-General framework}

\section{Quiescent crystallization}

Noting $x$ and $x_{\infty}$ the actual and ultimate degree of crystallinity, $\psi_{c}(T)\left(\right.$ resp. $\left.\psi_{a}(T)\right)$ the free energy for a purely crystalline (resp. purely amorphous) phase, $L$ the latent heat of crystallization, noting $\mathrm{n}($ resp. $\chi(\mathrm{T})$ ) an adjustable scalar (resp. function of temperature), we have shown in ${ }^{[1]}$ that Nakamura quiescent crystallization kinetics (1-2) can be put

\footnotetext{
${ }^{i} \underline{X}$ can be either a set of scalar, vector or tensor variables

$2 \partial \varphi^{*} / \partial \underline{Y}$ is a kind of generalization of the M matrix of the GENERIC formalism
} 
into the standard form (3) (4).

$$
\begin{gathered}
\alpha=\frac{x}{x_{\infty}}=1-\exp (-f) \\
\frac{d f}{d t}=\frac{n f^{n-1}}{\chi(T)} \\
\psi_{N}(T, f)=(1-\exp (-f)) x_{\infty} \psi_{c}(T)+\left(1-(1-\exp (-f)) x_{\infty}\right) \psi_{a}(T) \\
\Lambda=\rho_{0} \frac{\partial \psi_{N}}{\partial f}=\exp (-f) x_{\infty}\left(\psi_{a}(T)-\psi_{c}(T)\right)=\beta(T) \exp (-f) \approx L x_{\infty}\left(1-\frac{T}{T_{m}}\right) \exp (-f)
\end{gathered}
$$

and thus

$$
\frac{\partial \varphi_{N}^{*}(\Lambda)}{\partial \Lambda}=\frac{n}{\chi(T)}\left[\ln \left(\frac{\beta(T)}{\Lambda}\right)\right]^{1-\frac{1}{n}}
$$

\section{Flow induced crystallization}

A «natural» model for induced crystallization consists in simply adding the different potentials (index $\mathrm{M}$ referring to Mechanical constitutive behaviour and index $\mathrm{N}$ to Nakamura's kinetics). The coupling is achieved essentially by the fact that the mechanical parameters depend on the degree of crystallinity.

$$
\begin{aligned}
& \psi=\psi(T, f, \underline{\underline{c}})=\psi_{N}(T, f)+\psi_{M}^{(\hat{f}}(\underline{\underline{c}}) \\
& \varphi^{*}=\varphi_{N}^{*}(T, \Lambda)+\varphi_{M}^{*(\Lambda)}(\underline{z})
\end{aligned}
$$

Superscripts ${ }^{(f)}$ and ${ }^{(\Lambda)}$ mean that the rheological parameters (Maxwell relaxation time and viscosity for example) are parameterised by the degree of crystallinity. Governing equations are deduced from (6-7) in following the 3-abovementioned steps. In particular, kinetics of crystallization writes:

$$
\dot{f}=-\frac{\dot{\Lambda}}{\Lambda}=-\frac{\partial}{\partial \Lambda}\left(\varphi_{N}^{*}(T, \Lambda)+\varphi_{M}^{*(\Lambda)}(\underline{z})\right)
$$

\section{Flow induced crystallization for a power-law viscous fluid:}

A power-law viscous fluid is modelled here as a viscoelastic fluid undergoing very small elastic strains $\underline{\underline{\varepsilon}}_{\text {e }}$.

$\underline{\text { Step 1 : }}$

$$
\begin{aligned}
& \psi_{M}=\psi_{M}(T, \underline{\underline{\varepsilon}})=\frac{G}{2}\left(\underline{\underline{\varepsilon}}_{e}: \underline{\varepsilon}_{e}\right) \\
& \underline{\underline{Y}}=\frac{\partial \psi_{M}}{\partial \underline{\underline{\varepsilon}}_{e}}=G \underline{\underline{\varepsilon}}_{e}
\end{aligned}
$$

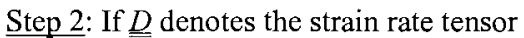




$$
\frac{\widetilde{D} \underline{\underline{\varepsilon}}_{e}}{\widetilde{D} t}=\frac{d \underline{\underline{\varepsilon}}_{e}}{d t}-\underline{\underline{D}} \approx-\underline{\underline{D}}
$$

The dissipation writes then

$$
\phi=\underline{\underline{\tau}} \underline{\underline{D}}-\underline{\underline{Y}}: \frac{d \underline{\underline{\varepsilon_{e}}}}{d t}=(\underline{\underline{\tau}}-\underline{\underline{Y}}): \underline{\underline{D}}-\underline{\underline{Y}}: \frac{\widetilde{\widetilde{D} \varepsilon_{e}}}{\widetilde{D} t}
$$

Leading to:

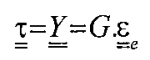

Step 3:

$$
\varphi_{M}^{*}(\tau)=\frac{m}{2^{m+1 / 2 m}(m+1) K^{1 / m}}\left(\tau^{d}: \underline{\tau}^{d}\right)^{m+1 / 2 m}
$$

Where $\underline{\underline{\tau}}^{d}$ denotes the deviator of $\underline{\underline{\tau}}\left(\tau_{i j}^{d}=-\tau_{k k} \delta_{i j}+\tau_{i j}\right)$

So that the constitutive law is classically written, in neglecting the elastic strains:

$$
\frac{\widetilde{D} \varepsilon_{e}}{\widetilde{D} t}=\frac{d \underline{\underline{\varepsilon_{e}}}}{d t}-\underline{\underline{D}}=-\frac{\partial \varphi_{M}^{*}}{\partial \underline{\underline{\tau}}} \quad \Rightarrow \quad \underline{\underline{\tau}} \quad \approx 2 K(2 \underline{\underline{D}}: \underline{\underline{D}})^{m-1 / 2} \underline{\underline{D}}
$$

Step 4: Assuming that $m$ is not affected by crystallization, equation (8) then writes:

$$
\frac{d f}{d t}=\frac{n}{\lambda(T)}\left[\ln \left(\frac{\beta(T)}{\Lambda}\right)\right]^{1-\frac{1}{n}}-\frac{m}{2^{m+1 / 2 m}(m+1)}\left(\tau^{d}: \tau^{d}\right)^{m+1 / 2 m} \frac{d}{d \Lambda}\left(\frac{1}{K(\Lambda)^{1 / m}}\right)
$$

\section{Discussion}

As a consequence of a kind of Onsager principle, the influence of flow on crystallization is completely determined by the influence of crystallization on the flow (i.e. by the way the rheological parameters depend on crystallinity through $\Lambda$ )

- In the Newtonian case $(m=1, K=\eta)$, the equations are explicit. As $\Lambda$ is an increasing function of the crystallinity $\alpha$, it can be seen that there is two mechanisms for crystallization. The first one has a thermal origin and is correctly described by the Nakamura law. The second one has a mechanical origin: as soon as the viscosity depends on $\Lambda$ and as the thermal crystallization is not instantaneous, there is a crystallization induced by the flow. This dependence is equivalent to a dependence of the viscosity on $\alpha$. An illustration of this point is found on two different rheological tests assumed to be carried out with polymers of different molecular length. In the first test we consider a shearing experiment carried out at a constant shear rate. The viscosity is proportional to the molecular length to a power of 3.4 , thus one can deduce from (15), that the longer the molecule is, the more effective is the flow induced crystallization. In a second test, we consider a shear experiment, which is carried out at a constant shear 
stress. The viscosity is then put at the denominator, which induces an opposite influence: the longer the molecule is, the less important is the induced crystallization.

- Figure 1 depicts the evolution of the shear viscosity with respect to time in a test carried out at a constant temperature and shear rate. The function $K(\Lambda)$ is adjusted to fit at best the experiments of Titomanlio etbbb al. ${ }^{[3]}$ (Figure 2). The qualitative evolution of viscosity with time is correctly predicted. It can also be noted from Figure 3 that, though no induction time has been artificially introduced into the model, an apparent induction time is correctly predicted: the crystallization seems to take place after a certain time only, and this induction time appears to be a decreasing function of strain rate.

\section{$\eta$ (Pa.s)}

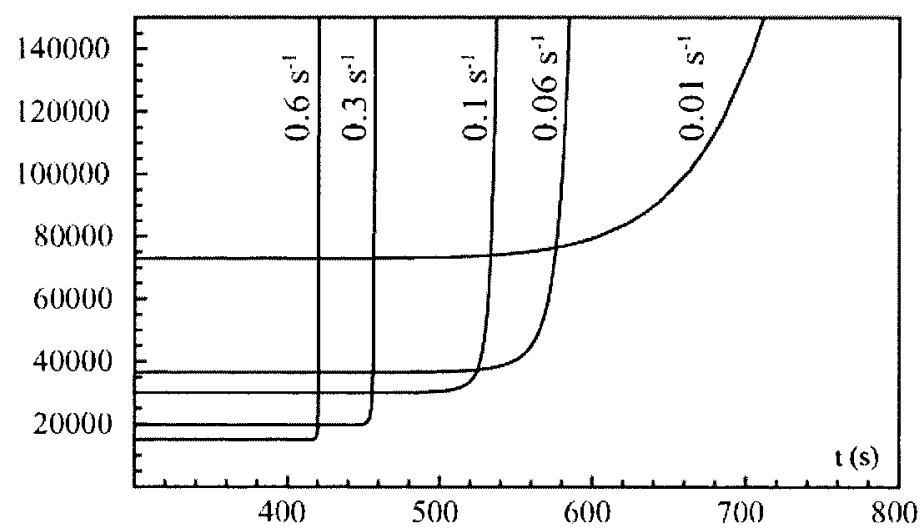

Figure 1 - Numerical predictions for increase of shear viscosity due to crystallization 


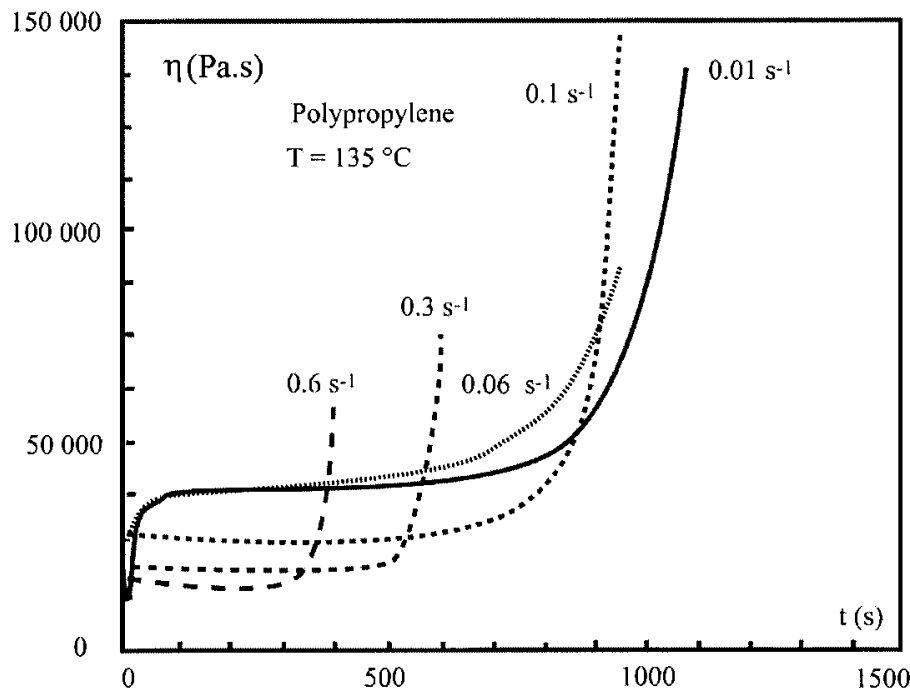

Figure 2 - Experimental increase of shear viscosity due to crystallization ${ }^{3}$

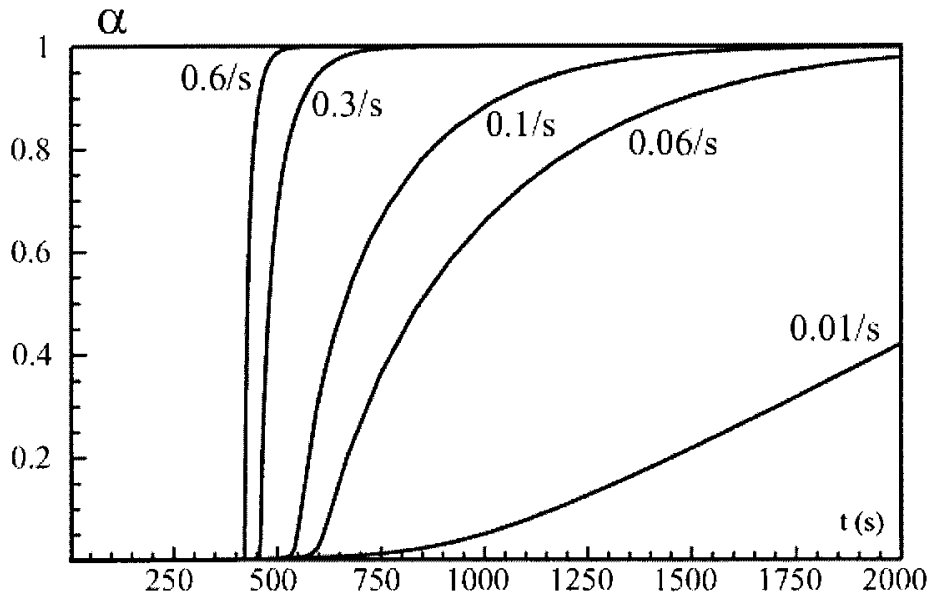

Figure 3 - Crystallinity induced by flow at different shear rates

\section{Flow induced Crystallization for a viscoelastic fluid}

\section{Upper convected Maxwell fluid}

If $\mathrm{K}$ denotes the elastic stiffness of the dumbbell, $v$ the number of chains per unit volume, $\mathrm{G}$ the elastic modulus of the Maxwell fluid and $\delta / \delta \mathrm{t}$ the upper convected

\footnotetext{
${ }^{3}$ Experimental results from Titomanlio et al. (1997)
} 
derivative, then the evolution of the conformation tensor $(\underline{\underline{c}}=<\underline{R} . \underline{R}>$ where $\underline{\mathrm{R}}$ denotes the end-to-end vector of the polymeric chain) is given by:

$$
\frac{\delta \underline{\underline{c}}}{\delta t}=-\frac{1}{\theta} \underline{\underline{c}}+\frac{G}{\theta v K}=
$$

Where $\delta / \delta$ t denotes the upper convected derivative

$$
\frac{\delta \underline{\underline{c}}}{\delta t}=\frac{d \underline{\underline{\underline{c}}}}{d t} \underline{\underline{\operatorname{grad}}} \underline{\underline{\underline{c}}} \underline{\underline{\underline{c}}}=\underline{\underline{\operatorname{grad}}} \underline{\underline{\underline{v}}}
$$

So that, if $\underline{\underline{D}}$ denotes the strain rate tensor, the extra stress tensor writes:

$$
\underline{\tau}=\nu K \underline{\underline{c}}-G \underline{\underline{1}}
$$

With

$$
\frac{\delta \tau}{\delta t}+\underline{\underline{\tau}}=2 \eta \underline{\underline{D}} \quad \text { and } \quad 2 \eta=G \theta
$$

Step 1: The free energy depends on temperature $\mathrm{T}$ and conformation tensor $\stackrel{c}{\underline{c}}$. So that, in neglecting thermo elasticity:

$$
\begin{gathered}
\rho_{0} \psi_{M}\left(\underline{\underline{c})}=\frac{1}{2} \mid \nu K \operatorname{Tr}(\underline{\underline{c})}-G \ln (\operatorname{det}(\underline{\underline{c}}))]\right. \\
\underline{\underline{z}}=\rho_{0} \frac{\partial \psi_{M}}{\partial \underline{\underline{c}}}=\frac{1}{2}\left[\nu K \underline{\underline{1}}-G \underline{\underline{\underline{c}}}{ }^{-1}\right]
\end{gathered}
$$

Step 2: For the upper convected Maxwell model, the derivative $\widetilde{D} / \widetilde{D} t$ is the upper convected one, so that a deformation is elastic if and only if $\delta c / \delta t=0$. The dissipation writes then:

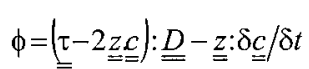

So that the expression for the extra stress tensor is deduced as:

$$
\tau=2 \underset{=}{z . c}
$$

Step 3: The pseudopotential is given by:

$$
\begin{array}{r}
\varphi_{M}^{*}(\underline{\underline{z}})=\frac{G}{2 \theta} \operatorname{lndet}\left(1-\frac{2}{\nu K} \underline{\underline{z}}\right)-\frac{G}{v K \theta} \operatorname{Tr}(\underline{\underline{z}}) \\
\frac{\widetilde{D} \underline{\underline{\underline{c}}}}{\widetilde{D} t}=\frac{\delta \underline{\underline{\underline{c}}}}{\delta t}=-\frac{\partial \varphi^{*}}{\partial \underline{\underline{z}}}=-\frac{1}{\theta} \underline{\underline{c}}+\frac{G}{\theta v K}=
\end{array}
$$

It is then straightforward to verify that equations (20) (22) (23) yield to the classical expression (19) for the upper convected Maxwell model

Step 4: In assuming that the relaxation time depends predominantly on crystallinity, equation (12) then writes: 


$$
\frac{d f}{d t}=\frac{n}{\lambda(T)}\left(\ln \left(\frac{\beta}{\Lambda}\right)\right)^{1-\frac{1}{n}}+\frac{\partial}{\partial \Lambda}\left(\frac{1}{\theta(\Lambda)}\right)\left(\frac{G}{2} \ln \operatorname{det}\left(1-\frac{2}{v K} \underline{z}\right)+\frac{G}{v K} \operatorname{Tr}(\underline{\underline{z}})\right)
$$

But

$$
\underline{\underline{z}}=\frac{1}{2} \vee K \underline{\underline{1}} \frac{1}{2} \vee K\left(\frac{\underline{\underline{\tau}}}{G}+1\right)^{-1}
$$

So that: $\frac{d f}{d t}=\frac{n}{\lambda(T)}\left(\ln \left(\frac{\beta}{\Lambda}\right)\right)^{1-\frac{1}{n}}-\frac{\partial}{\partial \Lambda}\left(\frac{1}{\theta(\Lambda)}\right)\left(\frac{G}{2} \operatorname{lndet}\left(\frac{\underline{\underline{\underline{\tau}}}}{G}+\frac{1}{=}\right)+\frac{G}{2} \operatorname{Tr}\left(\left(\frac{\underline{\underline{\tau}}}{G}+\frac{1}{\underline{1}}\right)^{-1}-3\right)\right)$

\section{Pom-Pom model}

The Pom-Pom model exhibits two relaxation mechanisms: tube orientation $\underline{\underline{S}}$ and backbone stretch $\lambda$. In its differential form, it can be written

$$
\begin{aligned}
& \underline{\underline{\tau}}=\frac{15}{4} G_{0} \phi_{b}^{2} \lambda^{2} \underline{\underline{S}} \\
& \frac{d \underline{\underline{S}}}{d t}=\underline{\underline{\operatorname{grad}}} \underline{\underline{S}} \cdot \underline{\underline{S}}+\underline{\underline{S}} \cdot \underline{\underline{\operatorname{grad} \nu^{T}}}-2(\underline{\underline{S}}: \underline{\underline{D}}) \underline{\underline{S}}-\frac{1}{\theta_{b}}\left(\underline{\underline{S}}-\frac{1}{3} \underline{1}\right) \\
& \frac{d \lambda}{d t}=\lambda\left(\underline{\underline{S}: D)}-\frac{1}{\theta_{s}}(\lambda-1)\right.
\end{aligned}
$$

$\underline{\text { Step } 1}$ : The free energy depends on $\underline{\underline{S}}$ and $\lambda$ and can be written, following Öttinger expression for the entropy ${ }^{[7]}$ :

$$
\rho \psi_{M}\left(T, \lambda, \underline{\underline{S})}=-\frac{1}{6} H \operatorname{lndet} \underline{\underline{S}}+\frac{1}{2} H\left(\lambda^{2}-2 \ln (\lambda)\right)\right.
$$

with

$$
H=\frac{15}{4} G_{0} \phi_{b}^{2}
$$

associated dual variables are

$$
\begin{aligned}
& \underline{y}=\rho_{0} \frac{\partial \Psi}{\partial \underline{\underline{S}}}=-\frac{1}{6} H \underline{\underline{S}}^{-1} \\
& \mu=\rho_{0} \frac{\partial \Psi}{\partial \lambda}=H\left(\lambda-\frac{1}{\lambda}\right)
\end{aligned}
$$

Step 2: The derivative $\widetilde{D} / \widetilde{D} t$ is defined by:

$$
\frac{\widetilde{D}}{\widetilde{D} t}\left(\begin{array}{l}
\underline{S} \\
\lambda
\end{array}\right)=\left(\begin{array}{l}
\frac{\Delta \underline{\underline{S}}}{\Delta t} \\
\frac{\tilde{\delta} \lambda}{\widetilde{\delta} t}
\end{array}\right)=\left(\begin{array}{c}
\frac{d \underline{\underline{\underline{S}}}}{d t}-\underline{\underline{\operatorname{grad}}} \underline{\underline{\underline{S}}}-\underline{\underline{S}} \cdot \underline{\underline{\operatorname{grad} v^{T}}}+2(\underline{\underline{S}: \underline{\underline{D}})} \underline{\underline{S}} \\
\frac{d \lambda}{d t}-\lambda \underline{\underline{\underline{S}: \underline{D})}}
\end{array}\right)
$$

$\Delta \Delta \mathrm{t}$ is a convective derivative preserving the trace, which is "naturally" associated to the upper convected derivative $\delta / \delta$ t for a tensor of trace unity $\left(\operatorname{Tr}(\underline{\underline{S}})=S_{k k}=1\right)$. More precisely:

$$
\frac{\Delta \underline{\underline{S}}}{\Delta t}=\frac{\delta \underline{\underline{S}}}{\delta t}-\operatorname{Tr}\left(\frac{\delta \underline{\underline{S}}}{\delta t}\right) \frac{\underline{S}}{\operatorname{Tr}(\underline{\underline{S}})} \quad \text { so that, } \quad \operatorname{Tr}\left(\frac{\Delta \underline{\underline{S}}}{\Delta t}\right)=0
$$

The dissipation writes then: 


$$
\phi=\underline{\underline{\tau}}: \underline{\underline{D}}-\underline{\underline{y}}: \frac{d \underline{\underline{S}}}{d t}-\mu \frac{d \lambda}{d t}
$$

Or, with equations (33), (34), (35)

$$
\phi=\left[\left(\underline{\underline{\tau}}-H \lambda^{2} \underline{\underline{S}}+\frac{H}{3} \underline{l}\right): \underline{\underline{D}}\right]-\underline{\underline{y}}: \frac{\Delta \underline{\underline{S}}}{\Delta t}-\mu \frac{\widetilde{\delta} \lambda}{\widetilde{\delta} t}
$$

But $\quad \frac{\Delta \underline{\underline{S}}}{\Delta t}=0 \quad$ and $\quad \frac{\widetilde{\delta} \lambda}{\widetilde{\delta} t}=0 \quad \Rightarrow \quad \phi=0$

Thus

$$
\underline{\underline{\tau}}=H \lambda^{2} \underline{\underline{S}}-\frac{H}{3} \underline{1}
$$

Step 3:

$$
\begin{aligned}
\varphi_{M}^{*}(\underline{\underline{y}, \mu}) & =\frac{1}{\theta_{b}}\left[-\frac{H}{6} \ln \left(\operatorname{det}\left(\frac{-2 \underline{\underline{y}}}{H}\right)\right)-\frac{1}{3} \operatorname{Tr}\left(\underline{\underline{y})}-\frac{H}{2}\right]\right. \\
+ & \frac{1}{\theta_{S}}\left[\int_{0}^{\mu}\left(\frac{m}{H}-1+\sqrt{1+\left(\frac{m}{H}\right)^{2}}\right) d m\right]
\end{aligned}
$$

It is then straightforward to verify that equations (33-35) and (41) yield the classical expression (28-30) for the Pom-Pom model.

Step 4: In assuming that the relaxation time depends predominantly on crystallinity, equation (12) then writes:

$\dot{f}=\frac{\partial}{\partial \Lambda}\left(\frac{1}{\theta_{b}(\Lambda)}\right)\left(-\frac{H}{6} \operatorname{lndet} \frac{-2 y}{H} \frac{1}{3} \operatorname{Tr}\left(\underline{\underline{y})}-\frac{H}{2}\right) \cdot \frac{\partial}{\partial \Lambda}\left(\frac{1}{\theta_{S}(\Lambda)}\right) \int^{\mu}\left(\frac{m}{H}-1+\sqrt{1+\left(\frac{m}{H}\right)^{2}}\right) d m+\frac{n}{\chi(T)}\left(\ln \left(\frac{\beta}{\Lambda}\right)\right)^{1-\frac{1}{n}}\right.$

\section{Discussion}

The most important point that we whish to discuss here are the following:

- As soon as we have chosen a model for the rheological behaviour of the polymer and that we are able to express it into the thermodynamical "standard" form, we are able to write a model for induced crystallization, which is "naturally" associated to this rheological model. A physical interpretation for this is that crystallization is related to the microscopic chain displacements and that the link between chain conformation and macroscopic mechanical is precisely the rheological constitutive relation. It can thus be understood that most of the induced crystallization mechanisms are already contained inside the rheology.

- This is not the only way to do it. McHugh et al. for example in a similar spirit (Generalized Hamiltonian Systems) decide to introduce a specific coupling function aimed to fit at best the crystallization kinetics. In case of necessity, we could do the same in our formalism, but we present here the simplest models. 
- This formalism allows one to achieve numerical simulations. Injection moulding is simulated for example with the Pom-Pom model. Numerical procedure is described in ${ }^{[8]}$. Figure 4 shows (for an advancing front coming from left side to right side), the evolution with time of the degree of crystallinity. At the end of the moulding stage (last picture), the skin layer begins to solidify and exhibits a crystallization affected by the flow. Figure 5 shows for the same experimental situation, the evolution of the stretch $\lambda$. Here again the skin layer is predominantly stretched. Figure 6 shows the orientation of the molecules. Near the flow front the fountain effects disorients the molecules, which are almost oriented near the walls.
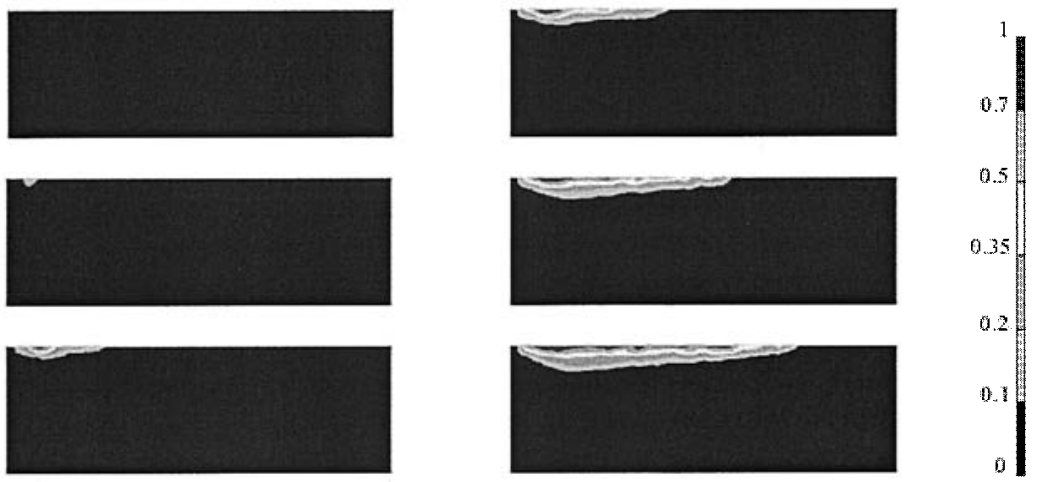

Figure 4 -Degree of crystallinity during the injection moulding process 

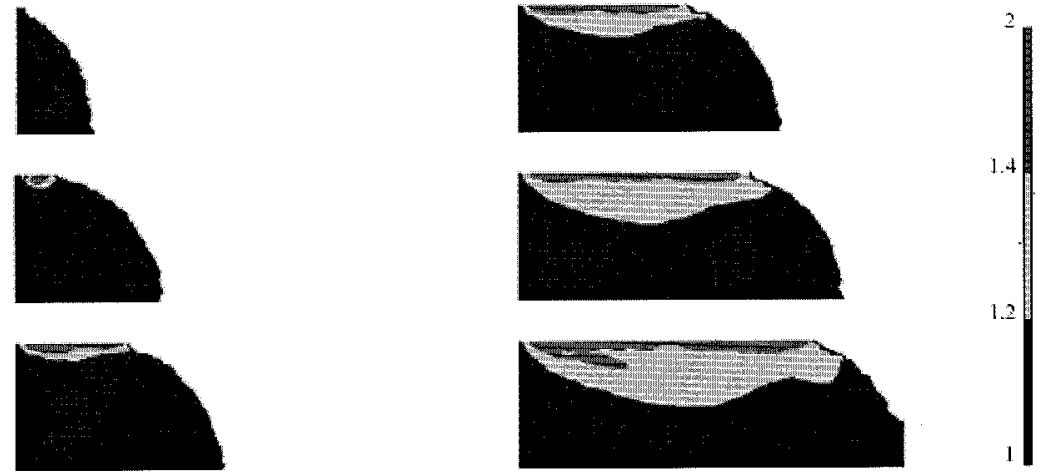

Figure 5 - Backbone stretch $\lambda$ in injection moulding
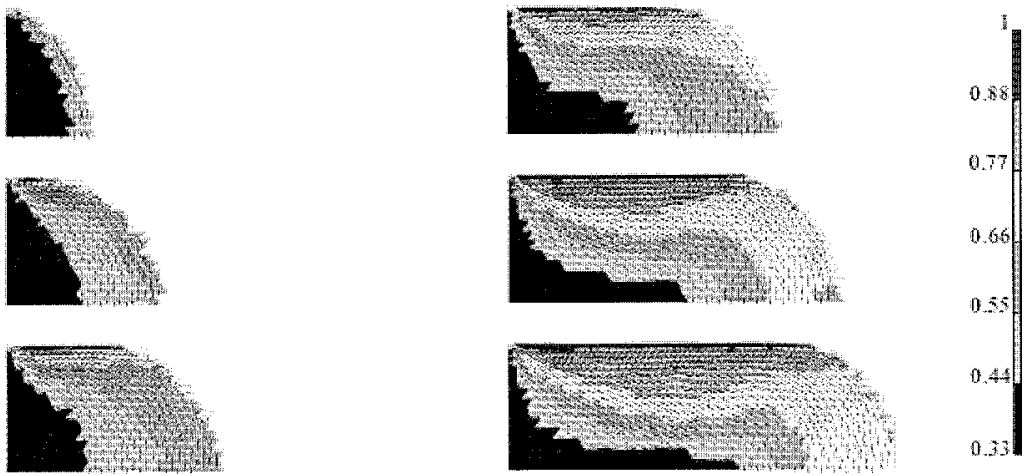

Figure 6 - Orientation tensor in injection moulding: arrows indicate the preferred orientation (eigenvector associated with the highest eigenvalue) and contours indicate $t$ level of this maximum eigenvalue 


\section{Conclusions}

We have presented in this paper a general framework for flow-induced crystallization. Much remains to do to reach a reasonable modelling. However we do believe that our formalism simplifies the physics because it does not exhibit to many adjustable parameters. Indeed, the only parameters are those that are related to the rheological characterization of the polymer with respect to the degree of crystallinity. There are obviously more phenomenological procedures, which are already able to model some specific processes, but none of them (to our knowledge) are able to deal with extension as well as with shear. This is one of the advantages of our approach.

[1] A. Poitou, A. Ammar. C. R. Acad. Sci. 2001, 329, 5.

[2] F. Jay, B. Monasse, J. M. Haudin. Int. J. of Form. Proc. 1998, 1, 75.

[3] G. Titomanlio, V. Speranza. Intern. Polym. Proc. 1997, 12, 45.

[4] S. Liedauer, G. Eder, H. Janeshitz-Kriegl. Intern. Polym. Proc. 1995, 10, 269.

[5] A. K. Doufas, I. S. Dairanieh, A. J. McHugh.. J. Rheol. 1999, 43, 85.

[6] M. Dressler, B. J. Edwards, H. C. Ottinger. Rheol. Acta. 1999 38, 117.

[7] H. C. Ottinger. XIIIth Intern. Congress on theology, Cambridge, UK 2000.

[8] A. Ammar, PhD Thesis, ENS de Cachan France 2001. 